\section{(- OPEN ACCESS}

\title{
Delayed neutrophil apoptosis enhances NET formation in cystic fibrosis
}

\author{
Robert D Gray, ${ }^{1}$ Gareth Hardisty, ${ }^{1}$ Kate H Regan, ${ }^{1}$ Maeve Smith, \\ Calum T Robb, ${ }^{1}$ Rodger Duffin, ${ }^{1}$ Annie Mackellar, ${ }^{1}$ Jennifer M Felton, ${ }^{1}$ Lily Paemka, ${ }^{2}$ \\ Brian N McCullagh, ${ }^{2}$ Christopher D Lucas, ${ }^{1}$ David A Dorward, ${ }^{1}$ Edward F McKone, ${ }^{3}$ \\ Gordon Cooke, ${ }^{4}$ Seamas C Donnelly, ${ }^{4}$ Pradeep K Singh, ${ }^{5}$ David A Stoltz, ${ }^{2}$ \\ Christopher Haslett, ${ }^{1}$ Paul B McCray, ${ }^{2}$ Moira K B Whyte, ${ }^{1}$ Adriano G Rossi, ${ }^{1}$ \\ Donald J Davidson ${ }^{1}$
}

\begin{abstract}
- Additional material is published online only. To view please visit the journal online (http://dx.doi.org/10.1136/ thoraxjnl-2017-210134).
\end{abstract}

${ }^{1} \mathrm{U}$ oE/MRC Centre for Inflammation Research, University of Edinburgh, Edinburgh, UK

${ }^{2}$ Carver College of Medicine, University of lowa, lowa City, lowa, USA

${ }^{3}$ Department of Respiratory Medicine, St Vincent's Hospital, Dublin, Ireland

${ }^{4}$ Department of Medicine, Trinity College Dublin and Tallaght Hospital, Dublin, Ireland

${ }^{5}$ Department of Microbiology, Washington University Medica School, Seattle, Washington, USA

\section{Correspondence to} Dr Robert D Gray, UoE/MRC Centre for Inflammation Research, University of Edinburgh, 47 Little France Crescent, Edinburgh EH16 4TJ, UK; r.d.gray@ed.ac.uk

$\mathrm{GH}$ and KHR contributed equally.

Received 13 February 2017 Revised 5 August 2017 Accepted 21 August 2017 Published Online First 15 September 2017

\section{ABSTRACT \\ Background Cystic fibrosis (CF) lung disease is} defined by large numbers of neutrophils and associated damaging products in the airway. Delayed neutrophil apoptosis is described in CF although it is unclear whether this is a primary neutrophil defect or a response to chronic inflammation. Increased levels of neutrophil extracellular traps (NETs) have been measured in CF and we aimed to investigate the causal relationship between these phenomena and their potential to serve as a driver of inflammation. We hypothesised that the delay in apoptosis in CF is a primary defect and preferentially allows CF neutrophils to form NETs, contributing to inflammation.

Methods Blood neutrophils were isolated from patients with CF, CF pigs and appropriate controls. Neutrophils were also obtained from patients with CF before and after commencing ivacaftor. Apoptosis was assessed by morphology and flow cytometry. NET formation was determined by fluorescent microscopy and DNA release assays. NET interaction with macrophages was examined by measuring cytokine generation with ELISA and qRTPCR.

Results CF neutrophils live longer due to decreased apoptosis. This was observed in both cystic fibrosis transmembrane conductance regulator (CFTR) null piglets and patients with $\mathrm{CF}$, and furthermore was reversed by ivacaftor (CFTR potentiator) in patients with gating (G551D) mutations. CF neutrophils formed more NETs and this was reversed by cyclin-dependent kinase inhibitor exposure. NETs provided a proinflammatory stimulus to macrophages, which was enhanced in CF. Conclusions CF neutrophils have a prosurvival phenotype that is associated with an absence of CFTR function and allows increased NET production, which can in turn induce inflammation. Augmenting neutrophil apoptosis in CF may allow more appropriate neutrophil disposal, decreasing NET formation and thus inflammation.

\section{CrossMark}

To cite: Gray RD, Hardisty G, Regan $\mathrm{KH}$, et al. Thorax 2018;73:134-144.

\section{INTRODUCTION}

Cystic fibrosis (CF) is the most common fatal single gene disorder in Caucasian populations with a prevalence of 1 in 2000 live births, and is a multiorgan disease affecting the lungs, pancreas, sweat glands, gut, liver and kidney. The inflammatory response to

\section{Key messages}

What is the key question?

- Do cystic fibrosis (CF) neutrophils live longer and allow the preferential formation of neutrophil extracellular traps (NET)?

What is the bottom line?

- CF neutrophils have a primary defect causing decreased spontaneous apoptosis and allowing increased levels of NET formation that can promote inflammation.

Why read on?

- This work links a fundamental cystic fibrosis transmembrane conductance regulatorrelated defect in neutrophil apoptosis to the promotion of inflammation in CF by NETs, and demonstrates that augmenting neutrophil apoptosis may be a therapeutic strategy to reduce NET formation and inflammation in CF.

infection with lower respiratory pathogens in $\mathrm{CF}$ is exaggerated with most patients dying from lung disease. ${ }^{1}$ The pathophysiology of CF lung disease is poorly understood but significant factors include impaired bacterial killing, decreased mucus clearance and overexuberant inflammation. ${ }^{2}$ Neutrophils are a key inflammatory cell in the CF lung acting as professional phagocytes, but their interaction with other immune cells in the lung may be as important and requires further investigation.

The ability of neutrophils to undergo spontaneous apoptosis is protective to the host and is central to the resolution of infectious or inflammatory insults. ${ }^{3}$ Several studies have demonstrated defects in apoptosis leading to increased neutrophil survival in $\mathrm{CF},{ }^{45}$ but the consequences of this remain unclear. In the absence of spontaneous apoptosis, other mechanisms of neutrophil disposal such as neutrophil extracellular trap (NET) formation (NETosis) may become increasingly important. NETosis has been proposed as an additional mechanism by which neutrophils can kill bacteria, functioning in addition to phagosomal killing. ${ }^{6}$ NETosis occurs in stimulated neutrophils (eg, by 
interleukin (IL)-8, lipopolysaccharide (LPS), phorbol myristate acetate (PMA) or bacteria) which undergo an oxidative burst and emit a network of DNA, histones, antibacterial and potentially proinflammatory proteins. An excess of NETs has been described in the CF airway, ${ }^{89}$ which could be due to either increased production or decreased clearance from the CF lung. NETs have also been associated with inflammatory conditions such as arthritis, systemic lupus erythematosus (SLE) and gout, ${ }^{10-12}$ and more recently NETs have been shown to interact with macrophages and prime inflammation in vascular disease. ${ }^{13}$ Therefore, the interaction of NETs with macrophages as a driver of inflammation could be particularly relevant in $\mathrm{CF}$, where macrophages have an overexaggerated response to inflammatory stimuli. ${ }^{14}$

We hypothesised that neutrophil survival is constitutively increased in CF due to decreased apoptosis, allowing more neutrophils to form NETs that will interact with macrophages and promote inflammation.

\section{METHODS}

\section{Collection of samples from patients with CF}

Peripheral blood was collected from stable patients with CF attending the Scottish National CF Service. Patients were considered stable if they had not required intravenous antibiotics in the past 2 weeks. Lung transplant patients were excluded. Patients gave written consent and the study was approved by regional ethics committees (East of Scotland Research Ethics Committee, 15/ES/0094, West of Scotland Research Ethics Committee, 11/ WS/0074). Anonymous matched healthy controls were recruited locally (Lothian Research Ethics Committee, 08/S1103/38). Sample collection from patients before and after ivacaftor therapy was approved by the St Vincent's University Hospital, Dublin, Research and Ethics Committee.

\section{Isolation of human neutrophils and peripheral blood mononuclear cells}

Human peripheral blood was collected into $3.8 \%$ sodium citrate. Plasma was aspirated following centrifugation of whole blood at $350 \times \mathrm{g}$ for $20 \mathrm{~min}$. Polymorphonuclear cells and peripheral blood mononuclear cells (PBMC) were isolated by $6 \%$ dextran sedimentation and separated by discontinuous $(72.9,63.0 \%$ and 49.5\%) Percoll (GE Healthcare, Buckinghamshire, UK) gradient as described. ${ }^{15}$ Isolated cells were washed twice in cation-free Dulbecco's phosphate buffered saline $\left(\mathrm{DPBS}^{--}\right)$and then resuspended in appropriate culture media. In some experiments (referred to in the Results section), neutrophils were isolated using Ficoll-Paque (GE Healthcare) dextran sedimentation and hypotonic lysis of residual erythrocytes.

\section{Cell viability and apoptosis measurement}

Isolated neutrophils $\left(5 \times 10^{6} / \mathrm{mL}\right)$ were cultured in 24 or 96 -well plates in Iscove's Modified Dulbecco's Medium (IMDM) (in some experiments Roswell Park Memorial Medium (RPMI) was substituted) supplemented with 5\% autologous serum (in some experiments $10 \%$ fetal calf serum (FCS) or no serum was substituted), $1 \%$ penicillin and streptomycin, and 1\% L-glutamine alone or in the presence of AT7519 (Astex Pharmaceuticals, Cambridge, UK), granulocyte-macrophage colony-stimulating factor (GM-CSF; R\&D Systems, Abingdon, UK) or E. coli LPS (Sigma, Dorset, UK) for 24 hours at $37^{\circ} \mathrm{C}, 5 \% \mathrm{CO}_{2}$. At stated time points, neutrophils were resuspended $(1: 5)$ in $\mathrm{DPBS}^{-/}$ supplemented with $25 \mathrm{mM}$ calcium chloride and labelled with Annexin V-FLUOS (Sigma) at 1:500 and $1 \mu \mathrm{g} / \mathrm{mL}$ propidium iodide (PI) before analysis on a BD FACS Scan, FACS Calibur or BD Accuri cell analyzer as described. ${ }^{16}$ Cytocentrifuge preparations were stained with Diff-Quick (Gamidor, Didcot, UK) to assess for morphological changes of apoptosis.

\section{Western blotting}

Western blotting was carried out as previously described, ${ }^{17}$ with the following antibodies: Mcl-1 (1:1000; Santa Cruz, Dallas, TX, USA), BAX (1:1000; Santa Cruz), $\beta$-actin (1:50 000; Sigma) and horseradish peroxidase-conjugated secondary antibodies (1:2500; Dako, Cambridgeshire, UK).

\section{Microscopic detection of NETs}

Neutrophils were seeded $\left(5 \times 10^{4} /\right.$ well) into 24 -well plates in RPMI with 5\% FCS, allowed to adhere for $30 \mathrm{~min}$ and then stimulated with $10 \mathrm{nM}$ PMA and incubated for 4 hours at $37^{\circ} \mathrm{C}$, $5 \% \mathrm{CO}_{2}$. In some experiments, they were allowed to adhere for 6 hours in the presence of media alone or media with $1 \mu \mathrm{M}$ AT7519 and/or $2.5 \mathrm{ng} / \mathrm{mL}$ GM-CSF prior to stimulation and further incubation for 4 hours. After the incubation time, $0.15 \mu \mathrm{M}$ SYTOX green (Invitrogen, Thermo Scientific, UK) was added before bright field and fluorescent $(470 / 22 \mathrm{~nm}$ light emitting diodes excitation) images were captured on an EVOS FL cell imaging system. NET formation was quantified as percentage of SYTOX positive NETs per $10 \times$ field (NETs \% of total cell count on bright field), as described. ${ }^{15}{ }^{18}$ All samples were plated in duplicate and multiple fields were counted per well.

\section{NET DNA release kinetic assay}

This was based on a published assay. ${ }^{15}{ }^{19}$ Isolated neutrophils were seeded $\left(5 \times 10^{4} /\right.$ well $)$ in RPMI 1640 media supplemented with 5\% FCS into a flat-bottom 96-well plate and allowed to adhere for $30 \mathrm{~min}$ at $37^{\circ} \mathrm{C}, 5 \% \mathrm{CO}_{2}$. NETs were induced by addition of $10 \mathrm{nM}$ PMA and detected at 30 min intervals in a Synergy HT BioTek plate reader by addition of $0.15 \mu \mathrm{M}$ SYTOX green, a cell permeable nucleic acid stain with excitation/emission spectra of 504/523 nm. In some experiments, $1 \mu \mathrm{M}$ AT7519 and/or $25 \mathrm{ng} / \mathrm{mL}$ GM-CSF were added to the culture media for 6 hours prior to PMA stimulation.

\section{Human macrophage culture}

Isolated PBMCs were seeded in IMDM into 6-well plates for 60 min (Nunc Upcell, ThermoFisher, Waltham, MA). Media was then removed, cells were washed twice in IMDM and remaining adherent monocytes were cultured in IMDM supplemented with $10 \%$ autologous serum, $1 \%$ penicillin and streptomycin, and $1 \%$ L-glutamine for 5 days at $37^{\circ} \mathrm{C}, 5 \% \mathrm{CO}_{2}$. On day 5 , cells were washed twice in $\mathrm{DPBS}^{-/}$before detachment. Monocyte-derived macrophages (MDM) were then seeded into 48-well tissue culture plates at $2.5 \times 10^{5} /$ well into IMDM supplemented with $10 \%$ autologous serum, $1 \%$ penicillin and streptomycin, and $1 \%$ L-glutamine for a further $1-2$ days at $37^{\circ} \mathrm{C}, 5 \% \mathrm{CO}_{2}$.

\section{Human NETs and macrophage coculture}

Isolated neutrophils were stimulated with $100 \mathrm{nM}$ PMA or dimethyl sulfoxide vehicle control in Hanks' balanced salt solution $\left(\mathrm{HBSS}^{-1}\right.$ ) for $15 \mathrm{~min}$ in rolling suspension at room temperature (to stop clumping). Neutrophils were then washed three times in $\mathrm{HBSS}^{-/-}$to remove any residual PMA before seeding $5 \times 10^{5} /$ well onto MDMs (2:1 neutrophil to MDM ratio) in $500 \mu \mathrm{L}$ IMDM supplemented with $10 \%$ macrophage-donor serum, $1 \%$ penicillin and streptomycin, and $1 \%$ L-glutamine for 24 hours at $37^{\circ} \mathrm{C}, 5 \% \mathrm{CO}_{2}$. PMA-treated neutrophils were considered NETing neutrophils (or NETs) and non-PMA-treated 
neutrophils as control neutrophils. Supernatants were collected at 24 hours, centrifuged at $300 \times g$ for $5 \mathrm{~min}$ to remove cell debris and frozen at $-80^{\circ} \mathrm{C}$. RNA was extracted from remaining adherent MDMs using the Direct-Zol RNA extraction kit per manufacturer's instructions (Zymo Research, Irvine, CA).

\section{Coculture supernatant ELISA}

IL-8 and tumour necrosis factor (TNF) were measured in culture supernatant using commercially available ELISAs (R\&D Systems) as per the manufacturer's instructions.

qRT-PCR of macrophage gene qRT-PCR was performed using commercially available TaqMan gene expression assays (ThermoFisher) for IL8, TNF, CXCL9, CCL17 and 18s RNA as per manufacturer's instructions. Data were expressed as fold change from control.

\section{Scanning electron microscopy of bronchial tissue}

Bronchial tissue was obtained from an explanted CF lung (under institutional board approval, University of Iowa) and fixed with $2.5 \%$ glutaraldehyde in $0.1 \mathrm{M}$ cacodylate buffer and processed for electron microscopy (EM). For full methods, see online supplementary data.

\section{Statistical analysis}

All data are expressed as mean \pm SEerror of the mean. Flow cytometry data were analysed using FlowJo software (TreeStar, Ashland, Oregon) or BD Accuri platform specific software. Data were analysed with GraphPad Prism (La Jolla, CA) by t-test, Mann-Whitey $U$ test or analysis of variance with appropriate post-test as noted in figures.

\section{RESULTS}

\section{CF neutrophils have increased survival due to less apoptosis}

CF neutrophils displayed increased survival when assessed by morphology on cytocentrifuge preparations (figure $1 \mathrm{~A}-\mathrm{C}$, $\mathrm{p}<0.001)$. We confirmed this using flow cytometry by Annexin $\mathrm{V}$ and PI staining. Control neutrophils displayed more completed apoptosis at 24 hours than CF neutrophils, (figure $1 \mathrm{D}, \mathrm{E}$, $\mathrm{p}<0.01$ ), and at 24 hours more neutrophils were viable in the CF samples suggesting a prosurvival phenotype secondary to a decrease in apoptosis (figure $1 \mathrm{~F}, \mathrm{p}=0.0004$ ). Pan-caspase inhibition effectively inhibited all cell death in CF neutrophils (figure $1 \mathrm{G}, \mathrm{p}<0.001$ ) confirming that apoptosis is the major mechanism of neutrophil death in CF but is delayed in comparison to healthy controls.

\section{Delayed neutrophil apoptosis in CF is related to a loss of cystic fibrosis transmembrane conductance regulator function}

Next we assessed whether the delayed apoptosis in CF was related to decreased cystic fibrosis transmembrane conductance regulator (CFTR) function in neutrophils. We obtained neutrophils (using Ficoll-Paque and red cell lysis) from 12 patients with at least one G551D mutation prior to and 2 days after commencing ivacaftor therapy (150 mg twice daily), a CFTR potentiator drug which increases conductance of abnormal CFTR and has a proven clinical effect. ${ }^{20}$ The samples were obtained as part of a previously published observational study, ${ }^{21} 22$ but prepared and analysed separately of the other work. Neutrophil survival at 24 hours was significantly decreased after ivacaftor therapy, suggesting that CFTR potentiation reverses the prosurvival phenotype in $\mathrm{CF}$ neutrophils (figure $2 \mathrm{~A}-\mathrm{C}, \mathrm{p}=0.014$ ). This effect was also seen when patients had treatment for 7 days (data available for six patients, online supplementary figure $S 1, p=0.0313)$. To ensure that the apoptosis inducing effects of ivacaftor were not a non-specific effect of the drug, we tested the ability of Ivacaftor to induce neutrophil apoptosis in healthy control neutrophils in culture. No effect was demonstrated after 20 hours of in vitro culture over a range of doses (online supplementary figure S2).

Next we investigated whether the neutrophil survival phenotype was related to the absence of CFTR in neutrophils by utilising CFTR null neutrophils from $\mathrm{CFTR}^{-/}$piglets (CF piglets). Neutrophils were harvested (using Ficoll-Paque and red cell lysis) from 2-week-old CF piglets and wild-type controls and cultured ex vivo in the presence of $10 \%$ FCS. CF piglet neutrophils demonstrated prolonged survival, suggesting that increased neutrophil survival (due to decreased apoptosis) is a primary defect related to absence of CFTR from neutrophils (figure 2D,E, $\mathrm{p}=0.027$ ).

\section{Prolonged CF neutrophil lifespan is not caused by inflammation}

Inflammation has been suggested to prolong neutrophil lifespan by inducing changes in the expression of Bcl-2 family members, in particular $\mathrm{Mcl}-1^{23}$ and $\mathrm{BAX} .^{5}$ Using samples from patients with $\mathrm{CF}$, we demonstrated no difference in the expression of Mcl-1 and BAX between CF neutrophils and healthy controls (figure $3 \mathrm{~A}-\mathrm{C}$ ), suggesting that inflammation-induced dysregulation of intrinsic apoptosis-regulating proteins was not the cause of the prolonged survival of CF neutrophils. Furthermore, the addition of prosurvival stimuli GM-CSF or LPS to CF neutrophils augmented the prosurvival phenotype, demonstrating that activating factors could further enhance the increased neutrophil survival in CF (figure $3 \mathrm{D}, \mathrm{p}<0.001$ ), rather than being the underlying mechanism. To assess whether a serum factor related to the inflammatory environment did not cause the apoptosis delay, we cultured healthy control neutrophils in the presence of CF serum. The addition of 5\% pooled CF serum to healthy neutrophil cultures did not increase neutrophil survival (figure 3E). Next we asked whether we could therapeutically reverse the apoptosis delay in CF neutrophils. Culture of CF neutrophils in the presence of AT5719, a cyclin-dependent kinase (CDK) inhibitor (CDKi) known to induce neutrophil apoptosis, ${ }^{17}$ significantly induced apoptosis in control $(p<0.01)$, and CF neutrophils $(\mathrm{p}<0.001)$, effectively correcting the apoptosis delay in CF to that of control levels (figure $3 \mathrm{~F}$ ).

\section{CF neutrophils form more NETs than healthy controls due to their prosurvival phenotype}

As CF neutrophils were characterised by reduced apoptosis, we then asked whether they were consequently more susceptible to NETosis. Freshly isolated human CF neutrophils formed NETs as efficiently as healthy control neutrophils in response to $10 \mathrm{~nm}$ PMA (figure 4A) after 4 hours of culture. This was confirmed by assessing NET formation by DNA release assay (figure 4B,E). However, DNA release from CF neutrophils diverged from control neutrophil DNA release at 6 hours, suggesting that $\mathrm{CF}$ neutrophils formed more NETs than non-CF controls as they aged in culture, and failed to engage in apoptosis (figure 4B). We therefore assessed whether aged CF neutrophils could form more NETs than aged non-CF control neutrophils. Neutrophils were aged for 6 hours and then stimulated with $10 \mathrm{nM}$ PMA to induce NETs. CF neutrophils formed significantly more NETs than similarly aged non-CF control neutrophils (figure 4C, $p=0.0087$, and figure 4D, $p<0.001)$. We then assessed whether either adding prosurvival factors to control neutrophils would 
A

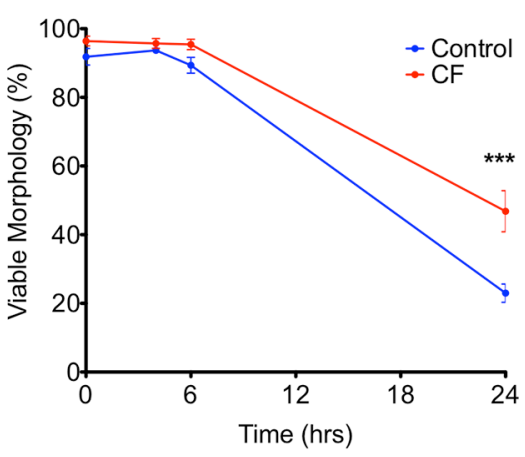

D

Control Flow $24 \mathrm{Hrs}$

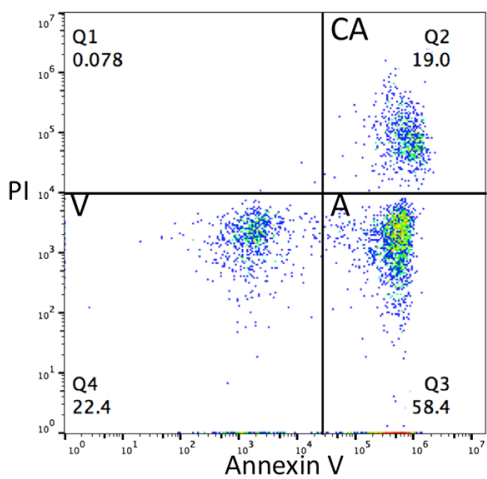

$\mathrm{F}$

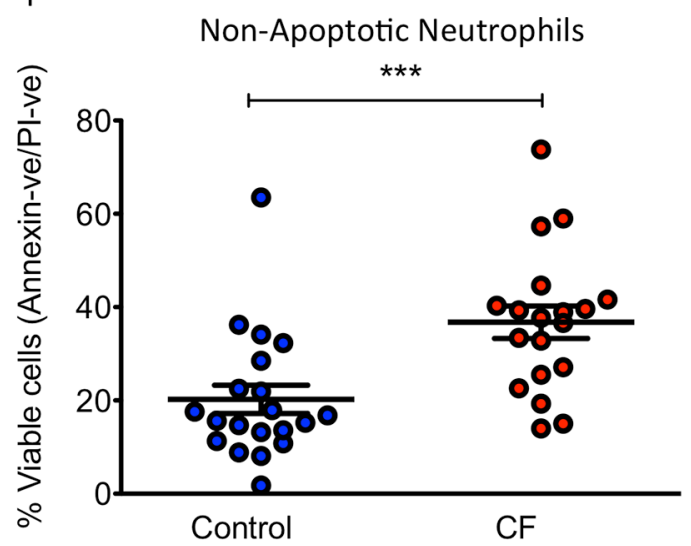

Viable

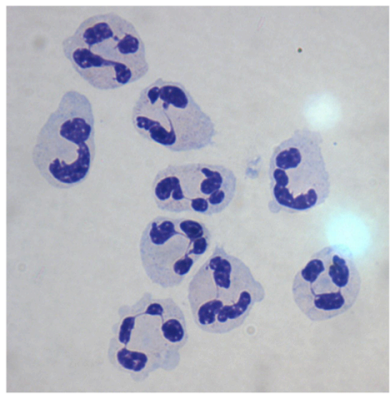

C

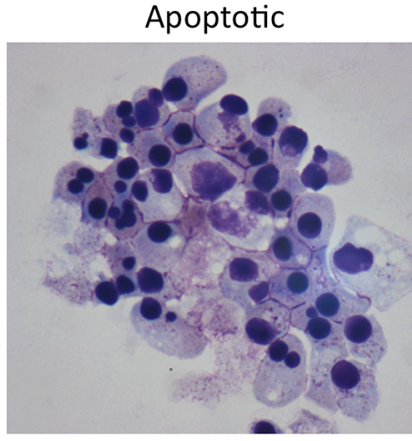

Figure 1 CF neutrophils have increased survival due to less apoptosis. (A) Cultured CF neutrophils are more viable at 24 hours than healthy controls when assessed by morphology ( $n=6$ CF, 5 controls). (B) Freshly isolated viable neutrophils in ex vivo culture demonstrating multilobed nuclei $(\times 100$ objective). (C) Apoptotic neutrophils following prolonged ex vivo culture demonstrating characteristic nuclear condensation ( $\times 100$ objective). (D) Flow plot of control neutrophils at 24 hours ex vivo culture demonstrating small numbers of viable cells ( $\mathrm{V}=$ annexin $\mathrm{V}$-ve/PI-ve cells) with the majority of cells having entered apoptosis ( $A=$ annexin $\mathrm{V}+\mathrm{ve} / \mathrm{PI}-\mathrm{ve}$ ) or completed apoptosis ( $\mathrm{CA}=$ annexin $\mathrm{V}+\mathrm{ve} / \mathrm{PI}+\mathrm{ve})$. ( $\mathrm{E}$ ) Control neutrophils have a higher rate of completed apoptosis than CF ( $n=19$ CF and 20 controls, for patient details see online supplementary table E1). (F) More viable neutrophils are present in CF culture at 24 hours ( $n=19$ CF and 20 controls). (G) Addition of the pan-caspase inhibitor Q-VD-OPh hydrate to CF neutrophil culture increases survival by inhibiting constitutive apoptosis. For patient details, see online supplementary table E1. Data presented as mean \pm SEM. Analysis with two-way analysis of variance and Bonferroni $(A, E, G)$, unpaired t-test (F). ${ }^{*} p<0.01$; ${ }^{* *} p<0.001$. CF, cystic fibrosis; PI, propidium iodide; QVD, Q-VD-OPh hydrate.

enhance NET formation, or inducing early apoptosis in CF neutrophils would reduce NET formation. The addition of the GM-CSF to non-CF control neutrophils for 6 hours prior to PMA stimulation increased NET formation (figure $5 \mathrm{~A}, \mathrm{p}<0.01$ ). Conversely, NET formation in CF neutrophils was inhibited by CDKi (figure 5A,B, $\mathrm{p}<0.001$ ).
NETs stimulate an inflammatory response from macrophages and this is exaggerated in CF

We used scanning EM to demonstrate the presence of NETs in the lungs of a patient with CF undergoing lung transplantation (figure 6A) based on morphology and features similar to previous descriptions of NETs on EM. ${ }^{9}$ Next we developed 

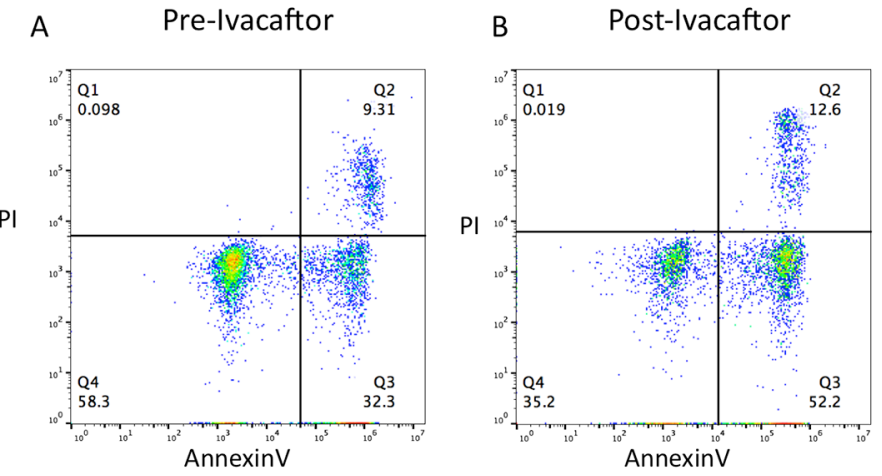

c

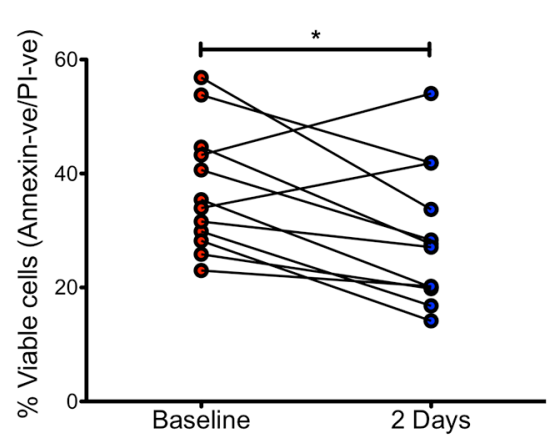

D

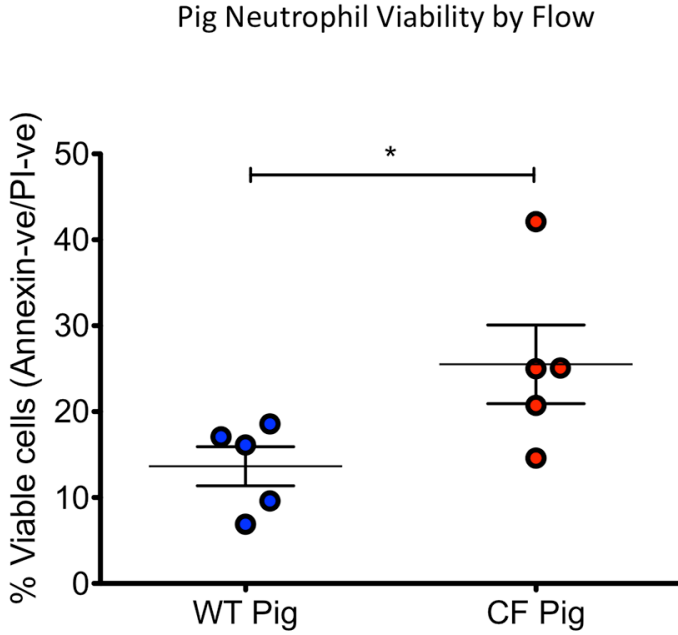

E

Pig Neutrophil Morphology

Baseline

WT Pig

CF Pig

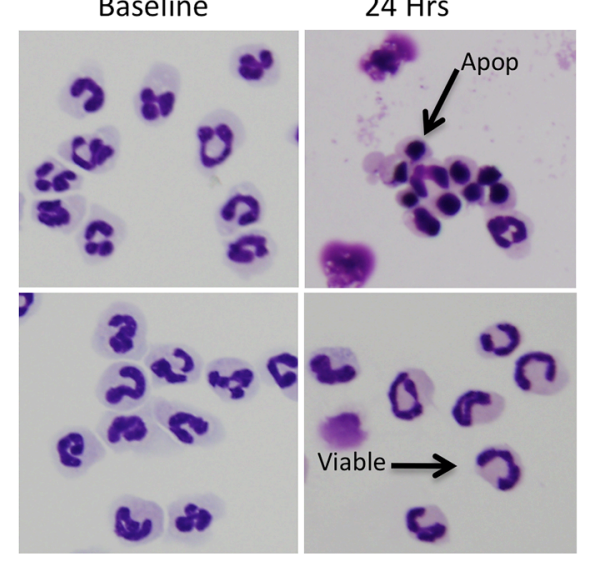

Figure 2 Delayed neutrophil apoptosis in CF is related to a loss of cystic fibrosis transmembrane conductance regulator (CFTR) function. Neutrophils were harvested from 12 patients with at least one G551D mutation before and after starting the CFTR potentiator ivacaftor (for patient details, see online supplementary table E2), and cultured ex vivo for 24 hours. (A) and (B) demonstrate example flow plots (following 24 hours ex vivo culture) from an individual patient before (A) and 2 days after (B) starting ivacaftor, showing that neutrophil survival decreased with treatment. (C) Combined data for 12 patients receiving ivacaftor, neutrophil viability decreased significantly with treatment. (D) Neutrophils were harvested from 2-week-old CF piglets and wild-type (WT) controls. Flow cytometry following 24 hours ex vivo culture demonstrated increased survival in CF (5 CF pigs and 5 WT controls). (E) Representative cytocentrifuge preparations of CF and WT neutrophils at 0 and 24 hours ( $\times 100$ objective) demonstrating increased numbers of apoptotic neutrophils in WTs at 24 hours. Data presented as mean \pm SEM. Analysis with paired t-test (C), Mann-Whitney U test (D). ${ }^{*} \mathrm{p}<0.05$. CF, cystic fibrosis; PI, propidium iodide.

a technique to enable the coculture of NETs (NETing neutrophils) and MDMs at consistent ratios by stimulating neutrophils with $100 \mathrm{nM}$ PMA under rolling conditions, washing and then adding them to MDMs where they then form NETs demonstrating classic morphology after 4-6hours (online supplementary figure S3). Supernatants and RNA were harvested from cocultures at 24 hours (figure 6B). The addition of NETs to healthy volunteer MDMs induced IL- 8 and TNF production (figure 6C,D, $\mathrm{p}<0.01$ and $\mathrm{p}<0.05$, respectively). This effect was also observed when NETs were added to CF MDMs (figure $6 \mathrm{C}, \mathrm{D}, \mathrm{p}<0.001$ both). The IL- 8 and TNF response to NETs was exaggerated in CF MDMs compared with healthy volunteers (figure $6 \mathrm{C}, \mathrm{D}, \mathrm{p}<0.05$ and $\mathrm{p}<0.001$, respectively). qRT-PCR confirmed increased IL-8 and TNF expression (not shown), and demonstrated that NETs induced the expression of CCL17, a marker of alternative activation in macrophages, ${ }^{24}$ in both CF and control MDMs (figure $6 \mathrm{E}$, $\mathrm{p}<0.001)$. Of note, CF MDMs had increased basal expression of the classical macrophage activation marker CXCL9 (figure 6F, p <0.001), ${ }^{25}$ which was further augmented by NETs (although without statistical significance). Overall, these data suggest that CF MDMs interacting with NETs have phenotypic markers of both classical and alternative activation.

\section{DISCUSSION}

Our data demonstrate that CF neutrophils survive longer due to delayed apoptosis and this is associated with the absence of CFTR function. Furthermore, this delay in apoptosis allows CF neutrophils to form NETs more efficiently than healthy control neutrophils, which may be an important proinflammatory mechanism. Impairment of neutrophil apoptosis in CF has been reported, ${ }^{426}$ and previous studies have suggested that this is either a response to systemic inflammation ${ }^{5}$ or, alternatively, a specific defect in CF neutrophils. ${ }^{426}$ The reversal of the apoptosis delay in patients with CF treated with (the CFTR potentiator) ivacaftor suggests that CFTR function in neutrophils is associated with apoptosis pathways. Indeed, previous studies have demonstrated direct effects of CFTR potentiation with ivacaftor on neutrophils, ${ }^{27} 28$ suggesting a fundamental role for CFTR in a number of neutrophil functions, consistent with CFTR being expressed at a biologically significant level. ${ }^{29}$ Our observation 


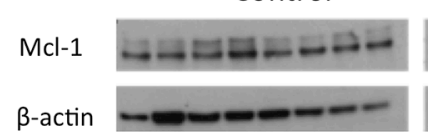

Control

C

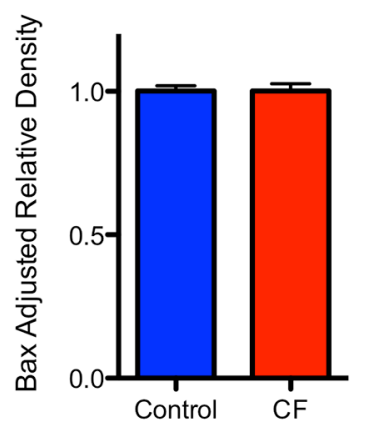

E

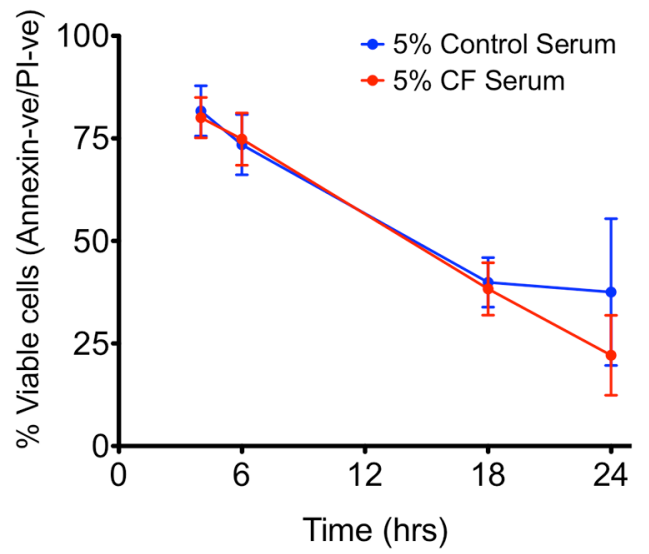

B

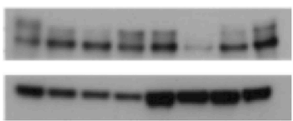

Control

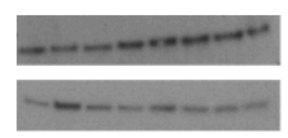

CF

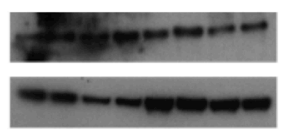

D

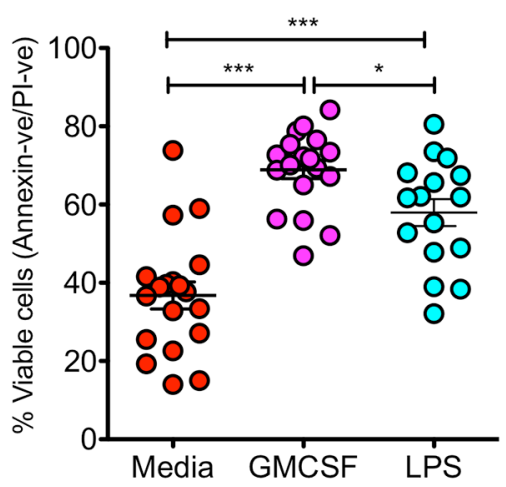

F

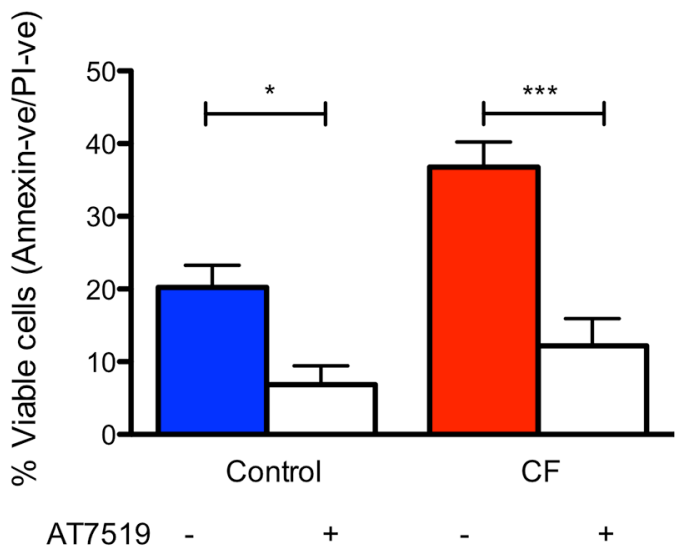

Figure 3 Prolonged CF neutrophil lifespan is not caused by inflammation. CF neutrophils have normal baseline apoptotic signalling of (A) Mcl-1, a $37 \mathrm{kDa}$ antiapoptotic protein, and (B) BAX, a $21 \mathrm{kDa}$ proapoptotic protein when freshly isolated ( $\mathrm{n}=8 \mathrm{CF}$ and 8 healthy controls, for patient details see online supplementary table E3). (C) Mcl-1 and BAX densitometry. (D) CF neutrophils retain sensitivity to delayed apoptosis with prosurvival stimuli GM-CSF $(20 \mathrm{ng} / \mathrm{mL})$ and LPS $(10 \mathrm{ng} / \mathrm{mL})$ for 24 hours ( $\mathrm{n}=19 \mathrm{CF}$ and 20 controls, for patient details see online supplementary table E1). (E) The primary apoptosis defect in CF is not due to a circulating CF serum factor. Healthy control neutrophils were cultured in media containing $5 \%$ pooled CF serum and this did not lead to increased neutrophil survival ( $n=3$ separate healthy donors). (F) The survival defect in CF could be corrected by culture with AT7519 $(1 \mu \mathrm{M})$ for 24 hours to augment neutrophil apoptosis and effectively reduce survival to healthy control levels ( $n=19 \mathrm{CF}$ and 20 controls, for patient details see online supplementary table E1). Data presented as mean $\pm S E M$. Analysis with unpaired t-test (C), one-way analysis of variance with Newman-Keuls post-test (D, F). ${ }^{*} \mathrm{p}<0.05 ;{ }^{* *} \mathrm{p}<0.01 ;{ }^{* * *} \mathrm{p}<0.001$. CF, cystic fibrosis; GMCSF, granulocyte-macrophage colony-stimulating factor; LPS, lipopolysaccharides; PI, propidium iodide.

that apoptosis is impaired in neutrophils from CFTR null piglets underlines the potential primary nature of delayed neutrophil apoptosis in CF. CFTR has been demonstrated to have a functional role in phagolysosomal activity in both macrophages ${ }^{30}$ and neutrophils, ${ }^{31} 32$ and the absence of CFTR promotes inflammatory cytokine release from macrophages. ${ }^{14} 33$ CFTR has also been implicated in the regulation of $\mathrm{T}$ cell suppression by myeloid suppressor cells ${ }^{34}$ and, as such, a diverse but functional role of CFTR in myeloid cells has now been established.

Our data demonstrate that the prolonged survival of $\mathrm{CF}$ neutrophils cannot entirely be explained by inflammation in CF. The Bcl-2 family pro and antiapoptotic proteins BAX and McL-1 have been implicated in inflammation-induced neutrophil survival. Mcl-1 excess is observed in neutrophils from patients with sepsis leading to prolonged neutrophil survival, ${ }^{23}$ but we demonstrated no difference in expression between CF and healthy control neutrophils. Inflammation-induced BAX deficiency has been suggested as a cause of delayed neutrophil apoptosis in CF and pneumonia, ${ }^{5}$ but again there was no difference in expression between CF and healthy control neutrophils. Our data infer that the apoptosis delay in CF is a primary feature of CF neutrophils and not simply related to inflammation, further emphasised by CF serum failing to prolong survival in healthy control neutrophils. Exposure of CF neutrophils to prosurvival stimuli such as GM-CSF or LPS further enhances survival, which may be particularly relevant once neutrophils have reached the inflammatory environment of the CF lung. The CF lung environment has been shown to induce changes in neutrophil behaviour and phenotype, ${ }^{35} 36$ and is therefore likely to accentuate the prosurvival phenotype that we demonstrate in CF. 
A

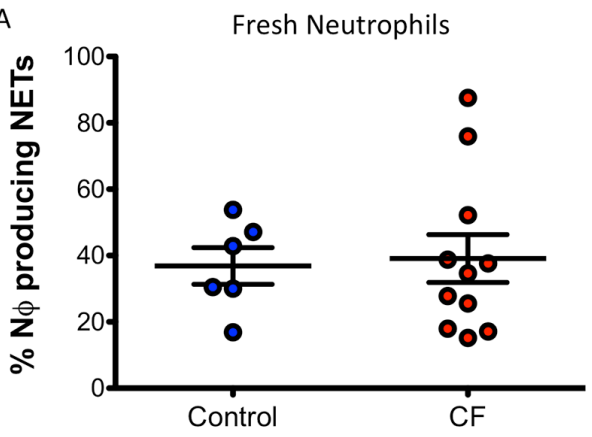

C

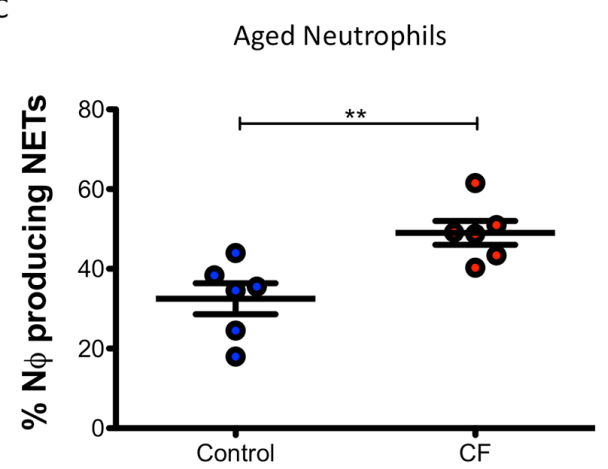

E
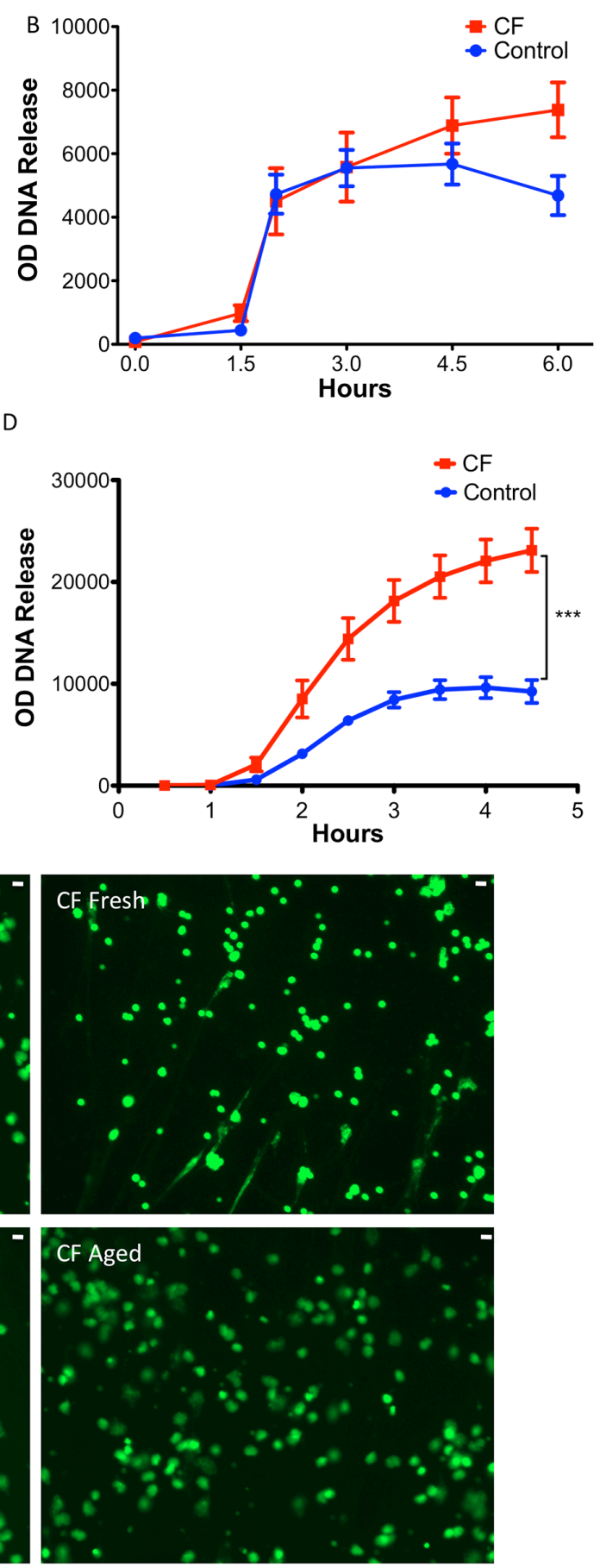

Figure 4 CF neutrophils form more NETs than healthy controls under conditions of ageing in culture. (A) Freshly isolated CF neutrophils form similar amounts of NETs as healthy controls (11 CF vs 6 controls, for patient details see online supplementary table E3) following stimulation with 10 nM phorbol myristate acetate (PMA). (B) DNA release as surrogate marker of NET production yielded similar results from PMA-treated freshly isolated neutrophils (7 CF vs 6 controls), although we observed a non-statistically significant increase in DNA release at later time points by CF neutrophils. (C) Neutrophils aged in culture for 6 hours prior to stimulation with PMA $(10 \mathrm{nM})$ demonstrated increased NET production by CF neutrophils versus controls ( $n=6$ CF vs 6 controls). (D) Increased NET production in aged neutrophils was confirmed by DNA release assay ( $n=6$ CF vs 6 controls). (E) Representative fluorescent microscopy following addition of SYTOX green in non-fixed cells, demonstrating increased PMA-induced NETs in CF following neutrophil ageing compared with controls (and also non-aged CF). Both diffuse morphology and spread morphology NETs are seen (scale bar $=10 \mu \mathrm{m})$. Data presented as mean \pm SEM. Analysis with unpaired t-test $(A, C)$, two-way analysis of variance with Bonferroni $(D)$. ${ }^{*} p<0.01$; ${ }^{* * *} p<0.001$. CF, cystic fibrosis; NET, neutrophil extracellular trap; OD, optical density.

We restored CF neutrophil apoptosis to healthy control levels by using the CDKi AT7519, a strategy that has been demonstrated to decrease inflammation in sterile and infective models of inflammation. ${ }^{17} 37$ Apoptosis is a well-described route of disposal for potentially toxic and damaging neutrophils and the phagocytosis of these apoptotic cells drives inflammation resolution. ${ }^{3}$ Targeting neutrophil apoptosis as an anti-inflammatory strategy in CF is therefore attractive, as it would offer 
A

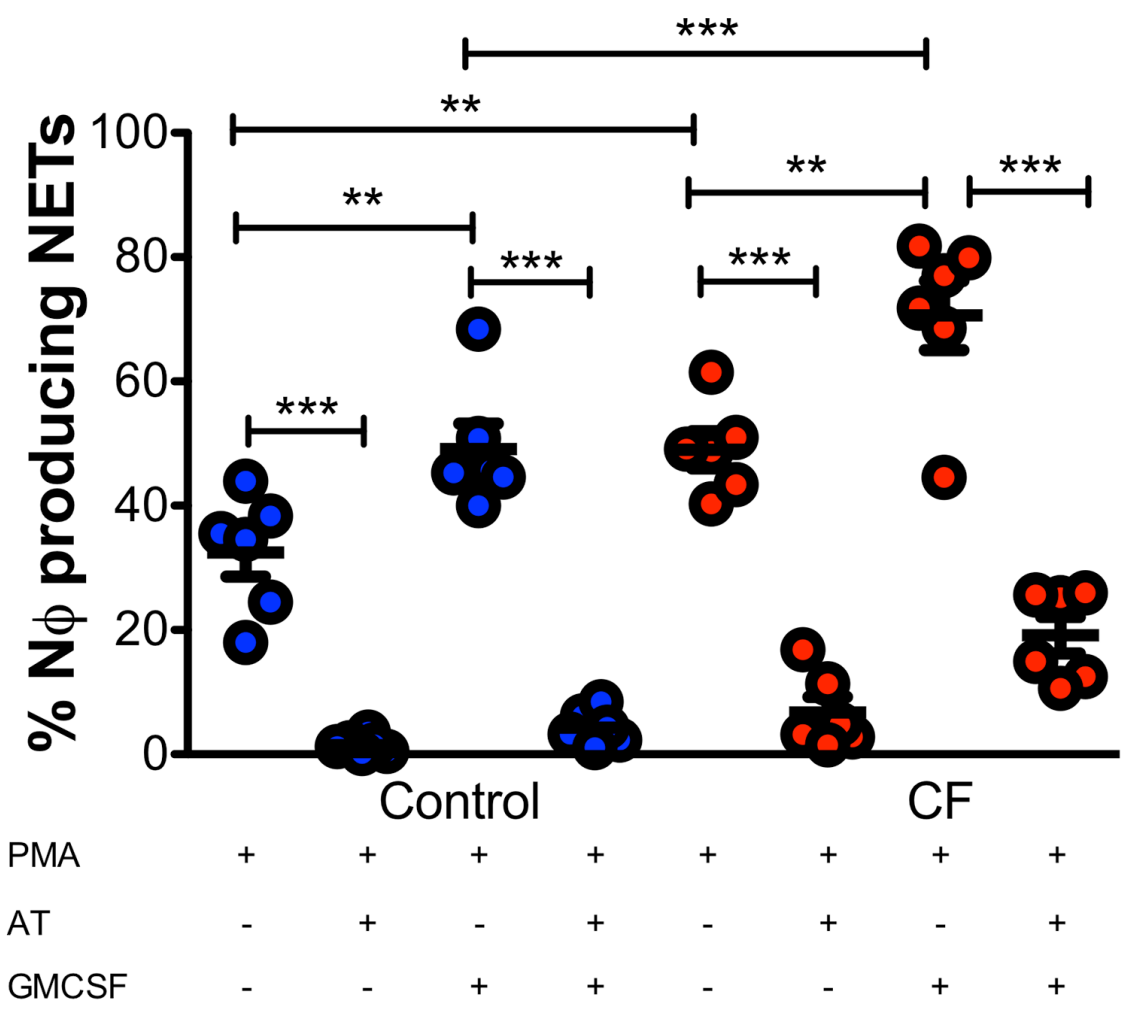

B
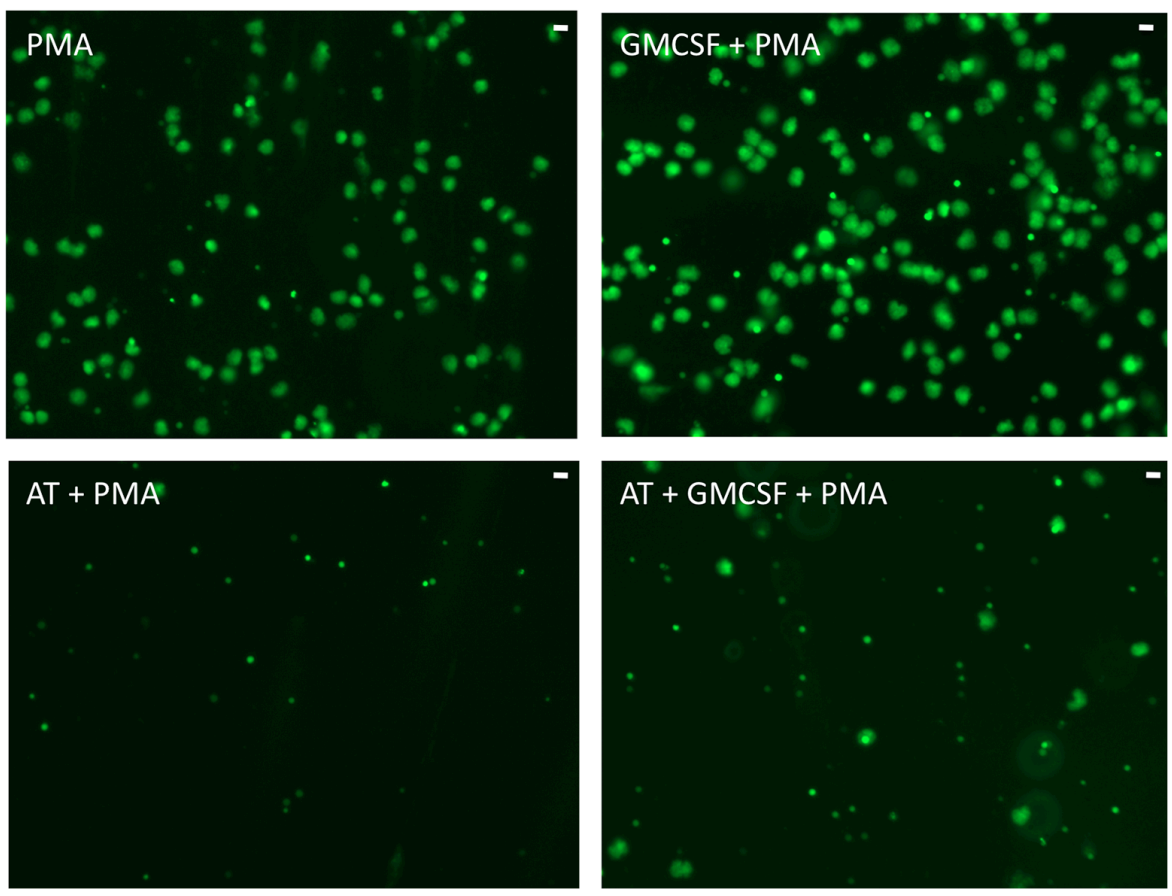

Figure 5 CF neutrophils form more NETs due to their prosurvival phenotype. (A) Healthy control and CF neutrophils were cultured for 6 hours in the presence of GM-CSF $(2.5 \mathrm{ng} / \mathrm{mL}) \pm A T 7519(1 \mu \mathrm{M})$ and then stimulated with PMA $(10 \mathrm{nM})$. The addition of GM-CSF to control neutrophils increased NET formation to that of CF levels. This effect was reversed by AT7519, suggesting that inducing apoptosis stopped NET formation ( $n=6$ CF and 6 healthy controls). (B) Representative fluorescent microscopic images showing SYTOX positive diffuse NETs in culture, enhanced by GM-CSF and inhibited by AT7519 in a patient with CF (scale bar=10 $\mu \mathrm{m})$. Data presented as mean \pm SEM. Analysis with one-way analysis of variance with Newman-Keuls posttest (A). ${ }^{* *} \mathrm{p}<0.01 ;{ }^{* *} \mathrm{p}<0.001$. CF, cystic fibrosis; GMCSF, granulocyte-macrophage colony-stimulating factor; NET, neutrophil extracellular trap; PMA, phorbol myristate acetate; AT, AT7519.

a universal therapy not dependent on a patient's individual CF genotype. Furthermore, engaging CF neutrophils in apoptosis more effectively may avoid more toxic forms of cell disposal such as NETosis.
We demonstrate for the first time that CF neutrophils form more NETs than healthy controls. Several lines of evidence highlight the importance of NETosis in CF. Excess amounts of DNA in the CF airway have been regarded as a potential contributor 
A

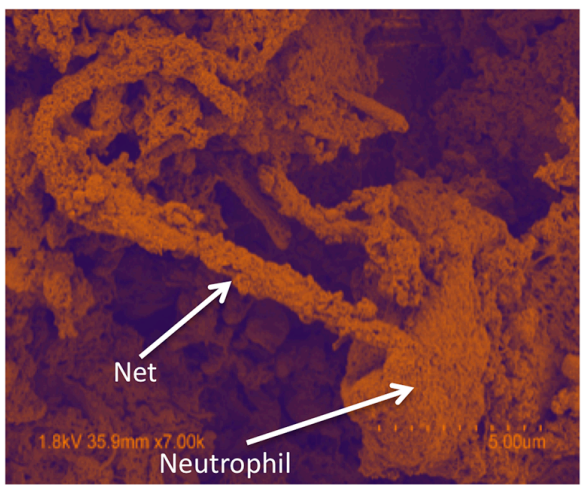

C

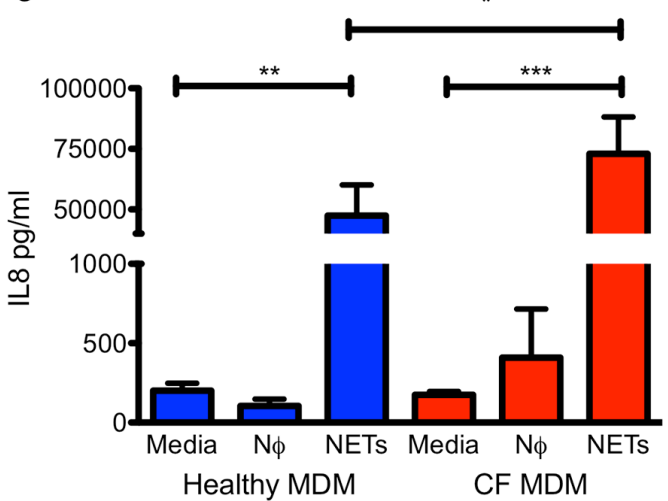

E

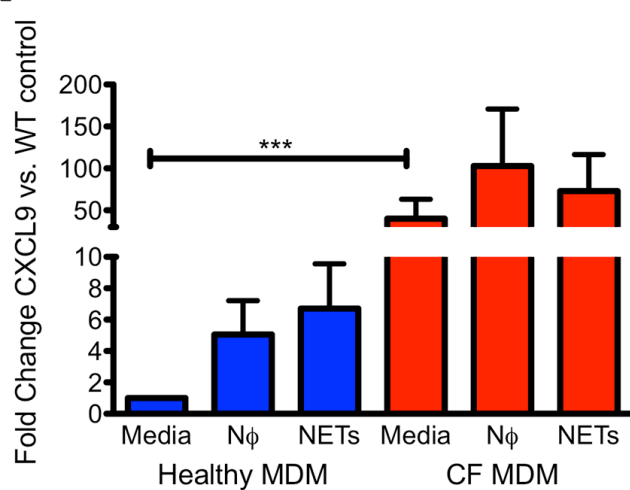

B

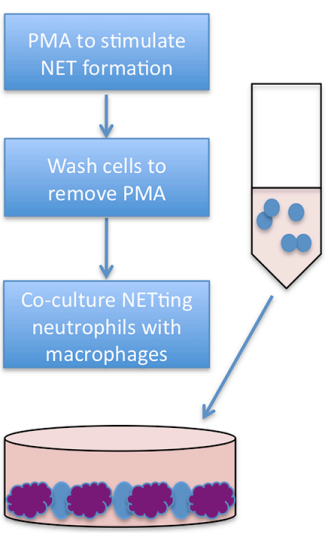

D

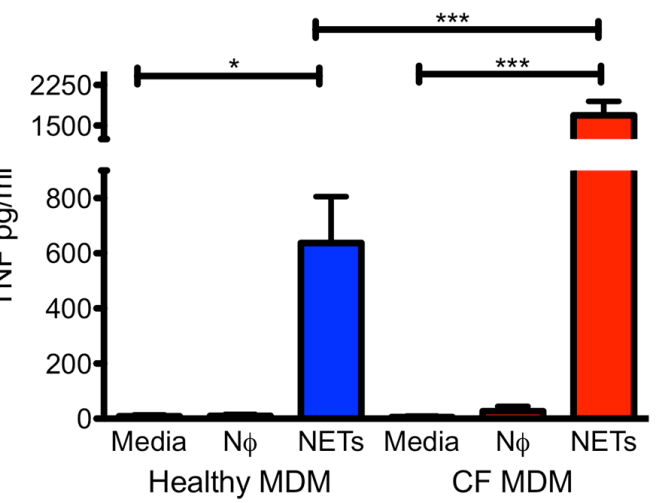

$\mathrm{F}$

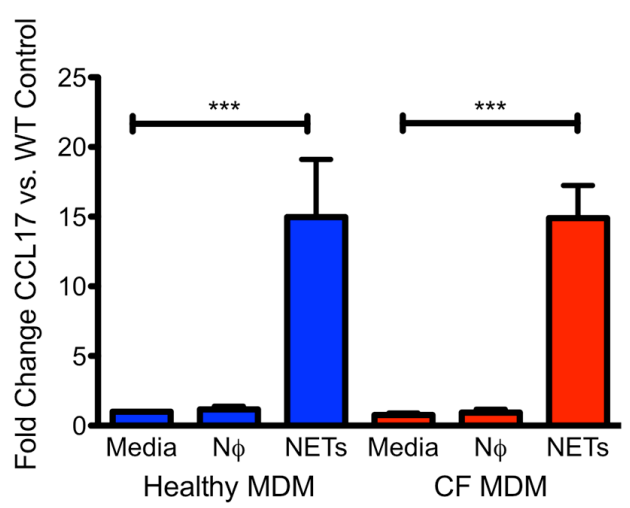

Figure 6 NETs stimulate an inflammatory response from macrophages and this is exaggerated in CF. (A) Scanning electron microscopy of bronchus from patient with explanted CF lung showing characteristic strands of NET-like material associated with neutrophils and bacteria. (B) Outline of novel NET/MDM coculture system. (C) NETs are proinflammatory to MDMs, causing an increase in IL-8 production after 24 hours of coculture and this effect is more pronounced with CF MDMs ( $n=8$ CF and 7 healthy MDM donors, for patient details see online supplementary table E3). (D) NETs induce TNF production from MDMs. (E) NETs induce CCL17 expression in healthy and CF MDMs. (F) NETs induce a non-significant increase in CXCL-9 expression in healthy controls, whereas CXCL-9 is overexpressed at baseline in CF. Data presented as mean \pm SEM. Analysis by one-way analysis of variance with Newman-Keuls post-test (C-F). ${ }^{* *} \mathrm{p}<0.01 ;{ }^{* * *} \mathrm{p}<0.001$. CCL, chemokine (C-C motif) ligand; $\mathrm{CF}$, cystic fibrosis; $\mathrm{CXCL}$, chemokine (C-X-C motif) ligand; IL, interleukin; MDM, monocyte-derived macrophage; NET, neutrophil extracellular trap; PMA, phorbol myristate acetate; TNF, tumour necrosis factor; WT, wild type.

to lung disease since the 1960s, although the presence of DNA in the form of NETs is a more recent finding. ${ }^{8} 93839$ These studies, however, fail to definitively address whether CF neutrophils form more NETs than healthy control cells. Our data demonstrate that CF neutrophils form more NETs than controls and this is directly related to the reduction in CF neutrophil apoptosis. The evidence for this is severalfold. First, the major difference in NET formation is observed when neutrophils are aged prior to PMA stimulation by which time control neutrophils, although viable, will have engaged early apoptotic machinery. Second, if non-CF neutrophils are cultured in the presence of GM-CSF before PMA stimulation (to delay apoptosis), they form equivalent numbers of NETs to CF neutrophils. And finally, culture of CF neutrophils for 6 hours in the presence of CDKi before PMA stimulation reduces the level of NET formation in CF to that of healthy controls. Taken together, these data 
suggest that CF neutrophils form more NETs because they are less able to engage in the normal process of apoptosis, but this can be reversed by augmenting apoptosis with CDKi, in keeping with other studies suggesting that the engagement of several non-apoptotic pathways is required for NET formation. ${ }^{40-42}$ These data also suggest that CDKi may offer a viable therapeutic strategy to subvert NETosis in CF and drive cells towards apoptosis and thus promote inflammation resolution. CDKs are present in a number of isoforms and have a diverse role in cell behaviour, although the major isoforms involved in the regulation of neutrophil apoptosis are CDKs 7 and $9 .{ }^{43} 44$ CDKi may also affect other kinases, for example, glycogen synthase kinase 3 (GSK-3), and in particular the GSK-3 $\beta$ isoform. ${ }^{45}$ Although it is worth noting that GSK-3 $\alpha$ is the major isoform found in neutrophils, ${ }^{46}$ this off-target effect may be less important in the context of the present work. Further study of the role of CDKs 7 and 9 in CF neutrophil apoptosis and NETosis will therefore be essential in our understanding of these processes.

There has been recent acknowledgement that granulocyte populations may display a degree of phenotypic heterogeneity leading to the description of low-density neutrophils (LDNs). ${ }^{47}$ Interestingly, a subclass of LDNs, low-density granulocytes (LDGs) may form greater numbers of NETs than 'normal' granulocytes in SLE, ${ }^{48}$ although the opposite findings have been described in the study of NETosis in rheumatoid arthritis. ${ }^{49}$ We did not investigate the role of LDNs in the present study, but the contribution of LDNs/LDGs to NET formation in CF would merit further investigation going forward.

We demonstrate that NETs are a potent proinflammatory signal to macrophages. The ability of NETs to kill bacteria has been described since their first description in 2004, ${ }^{6}$ but the potential inflammatory consequences of the presence of NETs in CF have not. We developed an assay to assess whether NETing neutrophils cultured at a consistent ratio with macrophages could promote the release of proinflammatory cytokines (from macrophages), and whether CF macrophages were more susceptible to this stimulus. NETs induced IL-8 and TNF release from MDMs in contrast to previous data. ${ }^{50}$ Our data are however consistent with the recent finding that NETs can prime macrophages for further cytokine release. ${ }^{13}$ We demonstrated an enhanced cytokine release from CF MDMs in response to NETs, consistent with CF macrophages being hyper-responsive to proinflammatory stimuli. ${ }^{14}$ We also demonstrate that the interaction of NETs with CF MDMs is associated with the expression of both classical and alternative markers of macrophage activation and would speculate that this could be a contributing factor to the non-resolving (frustrated) inflammation in CF. Although not addressed in this study, CF epithelial cells are a central part of the innate immune system in CF and as such the interaction of NETs with epithelial cells is of significant interest and will be subject to future study.

We acknowledge a number of limitations in this present study. First, we used peripheral blood neutrophils to study apoptosis and NETosis in the context of CF. Airway neutrophils may have a distinct phenotype in $\mathrm{CF}^{35}{ }^{36}$ and as such further study of the processes described in this paper (in airway neutrophils) may be indicated. Nevertheless, peripheral neutrophils have been used previously to assess neutrophil function, ${ }^{4} 27291$ underlining the validity of this approach. Second, we obtained neutrophils from CF adults with established lung disease and therefore established lung inflammation. An effect of inflammation on neutrophil death pathways in CF can therefore not be completely excluded by our data, although our data would suggest the mechanism is inflammation independent (particularly when we consider the results from $\mathrm{CFTR}^{-/-}$piglets). Further investigation of these processes in neutrophils from children with CF before and after the onset of significant inflammatory lung disease would help address this in the future. Third, although we suggest an association of CFTR deficiency with delayed apoptosis that is corrected by ivacaftor therapy in patients with the G551D mutation, further studies of CFTR potentiation and correction and its effects on neutrophil processes such as apoptosis and NETosis will be required. The advent of newer combination therapies to correct and potentiate the more common CF mutations such as F508del will make this possible in the future, ${ }^{52}$ allowing the study of both in vivo and in vitro correction of CFTR on neutrophil functions. Furthermore, we would suggest that the CFTR null pig may also be used to investigate neutrophil function and death related to CFTR deficiency, as an extension of our data in this paper and as recently studied in CF pig macrophages. ${ }^{33}$

In summary, we demonstrate that CF neutrophils have prolonged survival secondary to decreased apoptosis, and this is likely a primary defect in CF neutrophils. Increased NET formation by $\mathrm{CF}$ neutrophils is a consequence of a failure to engage in apoptosis and can be targeted by CDKi drugs. NETs are a powerful proinflammatory stimulus to macrophages, with CF-derived macrophages being hyper-responsive to NET stimulation. In conclusion, an intrinsic delay in neutrophil apoptosis enhances NET formation in CF and consequently inflammation, representing a novel target for the development of future anti-inflammatory therapies.

Acknowledgements We would like to thank the nursing staff of the Scottish National CF service for help with patient sample collection. We would also like to acknowledge use of the University of lowa Central Microscopy Research Facility and assistance with the electron microscopy partially supported by the Center for Gene Therapy for Cystic Fibrosis (NIH P30 DK-54759) and the Cystic Fibrosis Foundation. AT7519 was a kind gift from Astex Pharmaceuticals (Cambridge, UK).

Contributors RDG, GH, KHR, MS, CTR, RD, AM, JMF, LP, BNM, GC, CDL and DAD performed research. RDG, AGR, DJD, CH, MKBW, PKS, SCD, PBM, DAS and EFM designed the research. RDG, MKBW, AGR and DJD wrote the manuscript.

Funding This work was supported by the Wellcome Trust WT093767 (RDG)، WT094415 (CDL) and WT096497 (DAD); the UK Medical Research Council G1002046 (DJD) and MR/K013386/1 (AGR, RD, and CH). The studies in CF pigs were supported by NIH grants: P01 HL-51670, P01 HL-091842, and the Cystic Fibrosis Foundation RDP. Specimen collection from the patients receiving Ivacaftor was supported by an unrestricted investigator-initiated grant from Vertex Pharmaceuticals (Boston, MA, USA).

Competing interests None declared.

Ethics approval East of Scotland Research Ethics Committee, 15/ES/0094; West of Scotland Research Ethics Committee, 11/WS/0074; Lothian Research Ethics Committee, 08/51103/38.

Provenance and peer review Not commissioned; externally peer reviewed.

Open Access This is an Open Access article distributed in accordance with the terms of the Creative Commons Attribution (CC BY 4.0) license, which permits others to distribute, remix, adapt and build upon this work, for commercial use, provided the original work is properly cited. See: http://creativecommons.org/ licenses/by/4.0/

(c) Article author(s) (or their employer(s) unless otherwise stated in the text of the article) 2018. All rights reserved. No commercial use is permitted unless otherwise expressly granted.

\section{REFERENCES}

1 Morgan WJ, Butler SM, Johnson CA, et al. Epidemiologic study of cystic fibrosis: design and implementation of a prospective, multicenter, observational study of patients with cystic fibrosis in the U.S. and Canada. Pediatr Pulmonol 1999;28:231-41

2 Bartlett JA, Fischer AJ, McCray PB. Innate immune functions of the airway epithelium. Contrib Microbiol 2008; 15:147-63.

3 Jones HR, Robb CT, Perretti M, et al. The role of neutrophils in inflammation resolution. Semin Immunol 2016;28:137-45. 
4 Moriceau S, Lenoir G, Witko-Sarsat V. In cystic fibrosis homozygotes and heterozygotes, neutrophil apoptosis is delayed and modulated by diamide or roscovitine: evidence for an innate neutrophil disturbance. J Innate Immun 2010;2:260-6.

5 Dibbert B, Weber M, Nikolaizik WH, et al. Cytokine-mediated Bax deficiency and consequent delayed neutrophil apoptosis: a general mechanism to accumulate effector cells in inflammation. Proc Natl Acad Sci U S A 1999;96:13330-5.

6 Brinkmann V, Reichard U, Goosmann C, et al. Neutrophil extracellular traps kill bacteria. Science 2004;303:1532-5.

7 Brinkmann V, Zychlinsky A. Beneficial suicide: why neutrophils die to make NETs. Nat Rev Microbiol 2007;5:577-82.

8 Dwyer M, Shan Q, D'Ortona S, et al. Cystic fibrosis sputum DNA has NETosis characteristics and neutrophil extracellular trap release is regulated by macrophage migration-inhibitory factor. J Innate Immun 2014;6:765-79.

9 Manzenreiter R, Kienberger F, Marcos V, et al. Ultrastructural characterization of cystic fibrosis sputum using atomic force and scanning electron microscopy. J Cyst Fibros 2012;11:84-92.

10 Mitroulis I, Kambas K, Chrysanthopoulou A, et al. Neutrophil extracellular trap formation is associated with IL-1 $\beta$ and autophagy-related signaling in gout. PLoS One 2011;6:e29318

11 Khandpur R, Carmona-Rivera C, Vivekanandan-Giri A, et al. NETs are a source of citrullinated autoantigens and stimulate inflammatory responses in rheumatoid arthritis. Sci Trans/ Med 2013:5:178-40.

12 Garcia-Romo GS, Caielli S, Vega B, et al. Netting neutrophils are major inducers of type I IFN production in pediatric systemic lupus erythematosus. Sci Trans/ Med 2011:3:73-20.

13 Warnatsch A, loannou M, Wang Q, et al. Inflammation. Neutrophil extracellular traps license macrophages for cytokine production in atherosclerosis. Science 2015;349:316-20.

14 Zhang PX, Cheng J, Zou S, et al. Pharmacological modulation of the AKT/microRNA199a-5p/CAV1 pathway ameliorates cystic fibrosis lung hyper-inflammation. Nat Commun 2015;6:6221.

15 Gray RD, Lucas CD, MacKellar A, et al. Activation of conventional protein kinase C (PKC) is critical in the generation of human neutrophil extracellular traps. $J$ Inflamm 2013;10:12.

16 Lucas CD, Allen KC, Dorward DA, et al. Flavones induce neutrophil apoptosis by down-regulation of $\mathrm{Mcl}-1$ via a proteasomal-dependent pathway. Faseb $\mathrm{J}$ 2013;27:1084-94.

17 Lucas CD, Dorward DA, Tait MA, et al. Downregulation of Mcl-1 has anti-inflammatory pro-resolution effects and enhances bacterial clearance from the lung. Mucosal Immunol 2014;7:857-68.

18 Metzler KD, Fuchs TA, Nauseef WM, et al. Myeloperoxidase is required for neutrophil extracellular trap formation: implications for innate immunity. Blood 2011;117:953-9.

19 Jeffery U, Kimura K, Gray R, et al. Dogs cast NETs too: Canine neutrophil extracellular traps in health and immune-mediated hemolytic anemia. Vet Immunol Immunopathol 2015;168:262-8.

20 Ramsey BW, Davies J, McElvaney NG, et al. A CFTR potentiator in patients with cystic fibrosis and the G551D mutation. N Engl J Med 2011:365:1663-72.

21 Hisert KB, Schoenfelt KQ, Cooke G, et al. Ivacaftor-Induced Proteomic Changes Suggest Monocyte Defects May Contribute to the Pathogenesis of Cystic Fibrosis. Am J Respir Cell Mol Biol 2016;54:594-7.

22 Adam RJ, Hisert KB, Dodd JD, et al. Acute administration of ivacaftor to people with cystic fibrosis and a G551D-CFTR mutation reveals smooth muscle abnormalities. $\mathrm{JCl}$ Insight 2016;1:e86183.

23 Fotouhi-Ardakani N, Kebir DE, Pierre-Charles N, et al. Role for myeloid nuclear differentiation antigen in the regulation of neutrophil apoptosis during sepsis. Am J Respir Crit Care Med 2010;182:341-50.

24 Wirnsberger $\mathrm{G}$, Hebenstreit $\mathrm{D}$, Posselt $\mathrm{G}$, et al. IL-4 induces expression of TARC/CCL17 via two STAT6 binding sites. Eur J Immunol 2006:36:1882-91.

25 Martinez FO, Sica A, Mantovani A, et al. Macrophage activation and polarization. Front Biosci 2008;13:453-61

26 Moriceau S, Kantari C, Mocek J, et al. Coronin-1 is associated with neutrophil survival and is cleaved during apoptosis: potential implication in neutrophils from cystic fibrosis patients. J Immunol 2009;182:7254-63.

27 Pohl K, Hayes E, Keenan J, et al. A neutrophil intrinsic impairment affecting Rab27a and degranulation in cystic fibrosis is corrected by CFTR potentiator therapy. Blood 2014;124:999-1009.
28 Bratcher PE, Rowe SM, Reeves G, et al. Alterations in blood leukocytes of G551Dbearing cystic fibrosis patients undergoing treatment with ivacaftor. J Cyst Fibros 2016;15:67-73.

29 Painter RG, Bonvillain RW, Valentine VG, et al. The role of chloride anion and CFTR in killing of Pseudomonas aeruginosa by normal and CF neutrophils. J Leukoc Biol 2008:83:1345-53.

30 Di A, Brown ME, Deriy LV, et al. CFTR regulates phagosome acidification in macrophages and alters bactericidal activity. Nat Cell Biol 2006;8:933-44

$31 \mathrm{Ng} \mathrm{HP}$, Valentine VG, Wang G. CFTR targeting during activation of human neutrophils. J Leukoc Biol 2016;100:1413-24.

$32 \mathrm{Ng} \mathrm{HP}$, Zhou Y, Song K, et al. Neutrophil-mediated phagocytic host defense defect in myeloid Cftr-inactivated mice. PLoS One 2014;9:e106813.

33 Paemka L, McCullagh BN, Abou Alaiwa MH, et al. Monocyte derived macrophages from CF pigs exhibit increased inflammatory responses at birth. J Cyst Fibros 2017; 16:471-4.

34 Öz HH, Zhou B, Voss P, et al. Pseudomonas aeruginosa airway infection recruits and modulates neutrophilic Myeloid-Derived Suppressor Cells. Front Cell Infect Microbiol 2016:6:167.

35 Tirouvanziam R, Gernez Y, Conrad CK, et al. Profound functional and signaling changes in viable inflammatory neutrophils homing to cystic fibrosis airways. Proc Natl Acad Sci U S A 2008:105:4335-9.

36 Makam M, Diaz D, Laval J, et al. Activation of critical, host-induced, metabolic and stress pathways marks neutrophil entry into cystic fibrosis lungs. Proc Nat/ Acad Sci U SA 2009; 106:5779-83

37 Dorward DA, Felton JM, Robb CT, et al. The cyclin-dependent kinase inhibitor AT7519 accelerates neutrophil apoptosis in sepsis-related acute respiratory distress syndrome. Thorax 2017;72:182-5.

38 Young RL, Malcolm KC, Kret JE, et al. Neutrophil extracellular trap (NET)-mediated killing of Pseudomonas aeruginosa: evidence of acquired resistance within the CF airway, independent of CFTR. PLoS One 2011;6:e23637.

39 Papayannopoulos V, Staab D, Zychlinsky A. Neutrophil elastase enhances sputum solubilization in cystic fibrosis patients receiving DNase therapy. PLoS One 2011;6:e28526.

40 Remijsen Q, Kuijpers TW, Wirawan E, et al. Dying for a cause: NETosis, mechanisms behind an antimicrobial cell death modality. Cell Death Differ 2011;18:581-8.

41 Remijsen Q, Vanden Berghe T, Wirawan E, et al. Neutrophil extracellular trap cell death requires both autophagy and superoxide generation. Cell Res 2011:21:290-304

42 Itakura A, McCarty OJ. Pivotal role for the mTOR pathway in the formation of neutrophil extracellular traps via regulation of autophagy. Am J Physio/ Cell Physiol 2013;305:C348-54.

43 Leitch AE, Lucas CD, Marwick JA, et al. Cyclin-dependent kinases 7 and 9 specifically regulate neutrophil transcription and their inhibition drives apoptosis to promote resolution of inflammation. Cell Death Differ 2012;19:1950-61.

44 Wang K, Hampson P, Hazeldine J, et al. Cyclin-dependent kinase 9 activity regulates neutrophil spontaneous apoptosis. PLoS One 2012;7:e30128.

45 de la Torre AV, Junyent F, Folch J, et al. GSK3 $\beta$ inhibition is involved in the neuroprotective effects of cyclin-dependent kinase inhibitors in neurons. Pharmacol Res 2012;65:66-73.

46 Giambelluca MS, Cloutier N, Rollet-Labelle E, et al. Expression and regulation of glycogen synthase kinase 3 in human neutrophils. Int J Biochem Cell Biol 2013:45:2660-5

47 Scapini P, Marini O, Tecchio C, et al. Human neutrophils in the saga of cellular heterogeneity: insights and open questions. Immunol Rev 2016;273:48-60.

48 Villanueva E, Yalavarthi S, Berthier CC, et al. Netting neutrophils induce endothelial damage, infiltrate tissues, and expose immunostimulatory molecules in systemic lupus erythematosus. J Immunol 2011;187:538-52.

49 Wright HL, Makki FA, Moots RJ, et al. Low-density granulocytes: functionally distinct, immature neutrophils in rheumatoid arthritis with altered properties and defective TNF signalling. J Leukoc Biol 2017;101:599-611.

50 Farrera C, Fadeel B. Macrophage clearance of neutrophil extracellular traps is a silent process. J Immunol 2013;191:2647-56.

51 McKeon DJ, Condliffe AM, Cowburn AS, et al. Prolonged survival of neutrophils from patients with Delta F508 CFTR mutations. Thorax 2008;63:660-1.

52 Wainwright CE, Elborn JS, Ramsey BW, et al. Lumacaftor-Ivacaftor in Patients with Cystic Fibrosis Homozygous for Phe508del CFTR. N Engl J Med 2015;373:220-31. 